\title{
Vacunas anti-rotavirus: Al fin una realidad
}

\author{
MIGUEL O'RYAN G.
}

\section{New rotavirus vaccines: A reality at last}

Rotavirus is the most common cause of severe acute diarrhea among children less than 3 years of age. This infection causes 25 million medical visits, 2 million hospitalizations and 440.000 deaths every year worldwide. The development of rotavirus vaccines has followed a long and difficult path seriously hampered by the abrupt withdrawal of Rotashield® in 1999 due to its association with intestinal intussusception. After six years of intense research, the world celebrates the licensing of two new rotavirus vaccines, different in formulation and administration schemes albeit clearly safe and not associated with intussusception as demonstrated by large Phase III trials including more than 60.000 children. These vaccines, Rotarix ${ }^{\circledR}$ from Glaxo SmithKline Biologicals and Rotateq ${ }^{\circledR}$ from Merck Sharp \& Dohme, have demonstrated to be highly efficacious against severe rotavirus gastroenteritis caused by the most prevalent serotypes. Prompt incorporation of these new vaccines into the immunization programs of countries, especially those in the poorest areas of the world where these vaccines are most needed, will require joint efforts between governments, vaccine manufacturers, international and non-governmental organizations and foundations.

Key words: Rotavirus, vaccines, efficacy, safety, intussusception.

Palabras clave: Rotavirus, vacunas, eficacia, seguridad, intususcepción.

\section{Impacto de las infecciones gastrointestinales en el nuevo milenio}

Las infecciones gastrointestinales continúan siendo al año 2005 una significativa causa de morbilidad y mortalidad en el mundo. Si bien durante las últimas décadas se registra una declinación progresiva de la mortalidad asociada, especialmente en los países que demuestran un progreso global en su desarrollo socioeconómico, la incidencia de infección se ha mantenido relativamente constante en los últimos 25 años $^{1}$. Se estima que durante la década de los 90 ocurrieron 1,4 billones de episodios de infección gastrointestinal por año en niños bajo 5 años de edad, primordialmente en países en desarrollo, de los cuales 123.6 millones requirieron atención médica y 9 millones fueron hospitalizados. Aproximadamente 2 a 2,5 millones de niños mueren anualmente a causa de un síndrome diarreico agudo
(SDA) causado por una infección gastrointestinal, muertes que se concentran mayoritariamente en los países más pobres ${ }^{2,3}$. La mayor tasa de mortalidad ocurre en los lactantes más pequeños, especialmente bajo 1 año de edad (8,5 por 1.000/ año) ${ }^{3}$. Durante sus primeros 3 años de vida un niño chileno experimentará 2 a 3 episodios de SDA al año ${ }^{4}$. Si bien la mortalidad asociada a esta afección ha disminuido en forma importante en nuestro país, no sobrepasando en la actualidad una tasa de 2/100.000 habitantes, las más recientes estimaciones indican que el SDA determina 114.000 visitas médicas y 12.500 hospitalizaciones anuales en niños bajo los 3 años de edad ${ }^{5}$.

Actualmente se reconocen más de 20 microorganismos como causantes de SDA, considerando bacterias, virus y parásitos ${ }^{6}$. En la medida que el nivel global de desarrollo de un país mejora con el consiguiente progreso en nivel educacional e higiénico de la población y del ambiente,

Facultad de Medicina, Universidad de Chile, Instituto de Ciencias Biomédicas, Programa de Microbiología y Micología. 
disminuye la proporción de infecciones causadas por bacterias y parásitos y aumenta la proporción asociada a virus. Los virus que causan diarrea aguda se denominan virus entéricos, cuatro de los cuales tienen una asociación demostrada y universal con SDA: rotavirus, calicivirus humanos $(\mathrm{HuCV})$, astrovirus y adenovirus entéricos? La epidemiología de estos virus ha sido ampliamente estudiada en el concierto mundial. De estos estudios se puede concluir que en la mayoría de las regiones del mundo los virus entéricos en su conjunto causan aproximadamente $50 \%$ de los episodios de SDA no disentérico que ocurren en forma endémica (no asociado a brotes) en niños bajo 5 años de edad ${ }^{7,8}$.

\section{Impacto de la infección por rotavirus}

Los rotavirus son la causa más frecuente de diarrea asociada a vómito y/o fiebre en niños. La infección por rotavirus afecta fundamentalmente a niños bajo los 5 años, siendo el niño bajo 3 años el individuo en mayor riesgo de experimentar una infección sintomática, especialmente antes de los 24 meses de edad $^{9}$. Un niño puede tener 5 episodios de infección por rotavirus antes de cumplir los 5 años pero el primer episodio se asocia con frecuencia con mayor gravedad. Una primoinfección se podrá presentar con vómitos profusos, y/o diarrea líquida con o sin mucosidad, y/o fiebre que puede alcanzar a $40{ }^{\circ} \mathrm{C}$ o más, siendo la deshidratación el riesgo más significativo. La infección primaria puede causar sin embargo, un espectro de enfermedad que va desde infección asintomática (excreción viral sin síntomas) a un SDA leve, moderado o grave, e incluso, como se ha descrito más recientemente, cursar con viremia, lo que parece ocurrir frecuentemente en las infecciones por rotavirus ${ }^{10}$. Las reinfecciones tienden a ser en su mayoría más leves o asintomáticas, manifestándose por excreción viral en ausencia de síntomas?.

Las más recientes estimaciones indican que antes de su quinto cumpleaños uno de cada 5 niños requerirá una consulta médica, uno de cada 65 una hospitalización y uno de cada 293 morirá a causa de una gastroenteritis por rotavirus ${ }^{2}$. En cifras globales ello indica que anualmente en la población mundial menor de 5 años ocurrirá 25 millones de consultas, 2 millones de hospitalizaciones y 440.000 muertes asociadas a esta infección. El impacto de la infección, en términos de consultas médicas y hospitalizaciones, es similar entre los diferentes países, de manera independiente a su estado de desarrollo. Las muertes se concentran en los países más pobres ya que es en estas áreas donde existen serias falencias en el manejo de niños deshidratados por esta infección. Expresado de otra manera, un lactante que vive en Calcuta, India, en Santiago, Chile o en Barcelona, España, tendrá el mismo riesgo de infectarse por rotavirus y similar riesgo de requerir una consulta médica u hospitalización, pero el riesgo de morir por esta infección será muy superior en el lactante que vive en Calcuta.

En Chile, rotavirus es por lejos la causa más frecuente de SDA bajo 3 años de edad motivando cerca de $50 \%$ de las hospitalizaciones y $35 \%$ de las consultas de urgencia por SDA no disentérico ${ }^{5}$. Se estima por ello que anualmente en este grupo etáreo cerca de 58.000 y 8.000 niños bajo 3 años requerirán una consulta de urgencia u hospitalización respectivamente, a causa de esta infección. Un estudio en curso para determinar el impacto de la infección por rotavirus como causa de consulta ambulatoria sugiere que bajo 5 años de edad este agente se asocia con $\sim 11 \%$ de ellas, por lo que causaría aproximadamente 20.000 consultas anuales (O’Ryan M y Vallebuono C, comunicación personal, Proyecto MINSAL-Universidad de Chile).

Esta epidemiología, sumada a la falta de un tratamiento específico, ha sido la base para la recomendación de la OMS y otras instituciones mundiales de favorecer fuertemente la elaboración de una vacuna anti-rotavirus.

\section{Conocimientos virológicos, inmunológicos y de epidemiología molecular necesarios para comprender el desarrollo de vacunas}

Rotavirus humano fue descubierto en el año 1973 cuando fue se visualizado mediante microscopia electrónica en la biopsia intestinal de un niño australiano con diarrea aguda grave ${ }^{11}$. Los rotavirus están compuestos por una triple capa proteica que conforma esferas (cápsides) concéntricas (Figura 1). Al interior de la cápside interna (denominada también nucleocápside) se aloja el ARN compuesto por 11 segmentos de doble cadena y proteínas no estructurales. Los rotavirus que infectan al ser humano pertenecen en su gran mayoría (más del 95\%) al grupo A, clasificación determinada por la caracterización antigénica de la proteína VP6, la más abundante, y que conforma la cápside "intermedia" del virus. Los rotavirus de grupos D a F se han detectado exclusivamente en animales ${ }^{7}$. Rotavirus del grupo $\mathrm{B}$ y $\mathrm{C}$ causan, aunque con una frecuencia considerablemente menor, infección gastrointestinal en adultos y niños? 


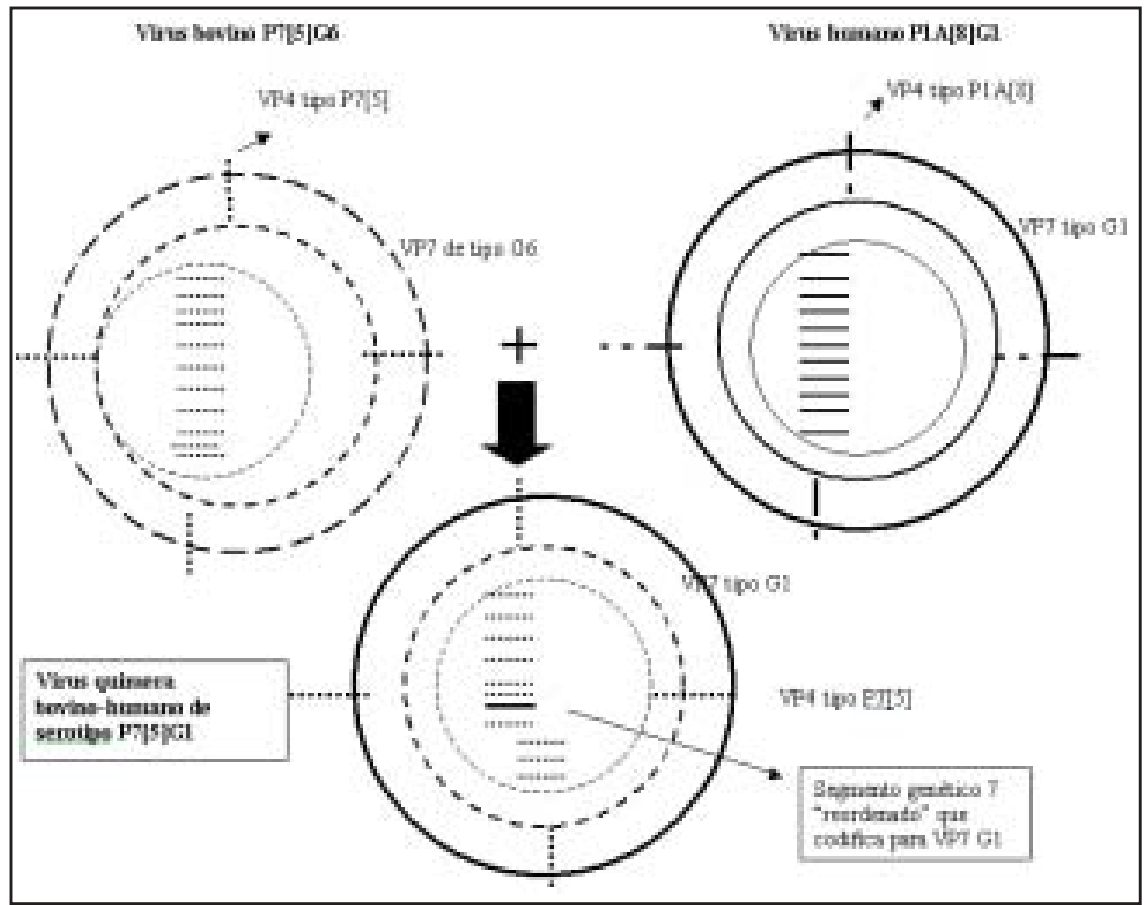

Figura 1. Esquema de reordenamiento de rotavirus bovino-humano.

Entre los rotavirus del grupo A se identifican diferentes tipos antigénicos, denominados serotipos, basado en diferencias antigénicas de las dos proteínas que conforman la cápside externa, VP7 y VP4 (Figura 1). VP7 es una glicoproteína (por lo que se le denomina proteína $\mathrm{G}$ ) que conforma la matriz de la cápside externa y VP4 es una proteína proteasa sensible (denominada por ello proteína P) que conforma las espigas que protruyen a través de la matriz. Se han descrito a la fecha 10 combinaciones antigénicas VP7/VP4 (o combinaciones G/P). La forma de descripción actualmente aceptada para los diferentes tipos de rotavirus es: serotipo VP4 [genotipo VP4] tipo VP7. A manera de ejemplo, el virus que se detecta con mayor frecuencia de tipo VP7 G1 y VP4 P1[8] se describe: P1A[8]G ${ }^{12}$.

La inmunidad anti-rotavirus ha sido objeto de un gran número de estudios in vitro, en animales, y clínicos durante los últimos 15 años. A pesar de ello, existen aspectos aún no esclarecidos de la inmunidad específica que no permiten tener una visión absolutamente clara de la interrelación entre los procesos inmunológicos involucrados en esta infección. Dos conceptos relevantes en que existe un cierto acuerdo son:

- La infección natural confiere protección contra reinfección, aunque dicha inmunidad no es completa, y es más efectiva contra reinfecciones sintomáticas ${ }^{9,13}$. Aunque rotavirus puede infectar a un mismo niño en varias ocasiones, después del primer episodio, que es frecuentemente sintomático, las infecciones subsecuentes son, en su mayoría, asintomáticas ${ }^{9}$. Es probable que las repetidas reexposiciones que ocurren a lo largo de la vida tengan un efecto booster, manteniendo activa la inmunidad y evitando así nuevos episodios de infección sintomática.

- VP7 y VP4 son los antígenos virales con capacidad de inducir anticuerpos neutralizantes. Éstos neutralizan el crecimiento del virus en líneas celulares y así mismo la infección sintomática en ratones expuestos al virus $^{12}$. En niños se ha correlacionado la presencia de anticuerpos séricos anti-VP7 con protección contra infección ${ }^{14-17}$. La protección tiende a ser completa si el individuo presenta altas concentraciones séricas de anticuerpos contra el VP7 que circula en su área geográfica (fenómeno denominado protección homotípica). Niños con altas concentraciones séricas de anti-VP7 expuestos a un rotavirus de serotipo diferente tendrán una protección parcial (protección heterotípica). Estos niños pueden infectarse aunque tienden a tener infecciones leves o asintomáticas. Es posible, aunque poco frecuente, que un niño tenga dos infecciones sintomáticas graves lo cual será más frecuente si el segundo virus es completamente diferente al primero. Como se verá 
más adelante, este concepto de protección homotípica versus heterotípica puede o no ser relevante para el desarrollo de vacunas.

Estudios de epidemiología molecular han permitido determinar que cuatro combinaciones de tipos G/P: P1A[8]G1, P1B[4]G2, P1A[8]G3, $\mathrm{P} 1 \mathrm{~A}[8] \mathrm{G} 4$ representan más del $95 \%$ de los tipos circulantes en todo el mundo y de ellas P1A[8]G1 es el serotipo que se detecta con mayor frecuencia globalmente. Recientemente el tipo P1A[8]G9 ha cobrado importancia epidemiológica en varias regiones del mundo incluyendo Latinoamérica, Australia y Europa ${ }^{18-21}$. Dos o más serotipos pueden co-circular en una misma ciudad durante un período determinado, aunque lo frecuente es que predominen sólo uno o dos serotipos ${ }^{13,18,22}$. El (los) serotipo(s) prevalente(s) en un área puede(n) cambiar de un año a otro probablemente en relación al status de seroprotección contra serotipos específicos de la población en el momento de introducirse un "nuevo" serotipo. Se ha descrito la circulación de serotipos menos comunes, P2A[6]G9 en India, virus con VP4 propios de cepas animales en Bangladesh, cepas P1A[8]G5, $\mathrm{G} 8$, y G10 en Brasil ${ }^{18,19,23,24}$. Estos nuevos serotipos probablemente surgen del reordenamiento (reassortment en inglés) natural de genes de rotavirus humanos y animales que ocurre en intestinos de animales co-infectados con más de un rotavirus (en la Figura 1 se aprecia un ejemplo de reordenamiento). Una observación intrigante ha sido la identificación de serotipos "poco comunes" en unidades de recién nacidos, algunas de las cuales se han asociado con infección asintomática ${ }^{18,25-27}$. Estas cepas podrían ser "naturalmente atenuadas" y han sido consideradas como candidatos para elaborar vacuna.

\section{Vacunas anti-rotavirus}

\section{Estrategias de desarrollo}

La comprobación clínico experimental de que un episodio de infección natural en niños confería protección contra nuevas infecciones, fue la base que estimuló los esfuerzos encaminados a producir una vacuna anti-rotavirus ${ }^{28}$. Las estrategias de desarrollo de vacunas han sido fundamentalmente dos. La primera basada en el uso de una sola cepa de rotavirus (denominada vacuna monovalente) obtenida de animales o humanos, y la segunda basada en el uso de más de una cepa de rotavirus (vacuna multivalente) que incluye varios virus de diferentes serotipos producto de reordenamientos entre virus animales y humanos (Tabla 1) $)^{9-36}$.

Las vacunas monovalentes animales se basan

Tabla 1. Vacunas anti-rotavirus candidatas

\begin{tabular}{lllll}
\hline Cepa & Serotipo(s) & Institución * & Origen de cepa & Etapa de desarrollo \\
\hline $\begin{array}{llll}\text { Virus animales } \\
\text { LLR }\end{array}$ & P[12]G10 & Inst. Lanzhou, China & Ovejuno & Licenciada en China \\
NCDV & P6[1]G6 & SmithKline & Bovino & Detenido \\
WC3 & P7[5]G6 & SmithKline & Bovino & Detenido \\
RRV & P5B[3]G3 & NIH & Simio & Detenido \\
Virus humanos & & & & \\
85-12 & P1A[8]G1 & GSK Rotarix ${ }^{\circledR}$ & Humana atenuada & Licenciada \\
RV3 & P2A[6]G3 & U. Melbourne & Neonatal & Fase II \\
$116 E$ & P8 [11]G9 & Bharat Biotech, India & Neonatal & Fase I \\
I-E32 & P8[11]G10 & Bharat Biotech, India & Neonatal & Fase I \\
M37 & P[3]G1 & NIH & Neonatal & Detenido \\
Virus reordenados & & & & \\
RRV-humano & G1-G4, P5B[3] & Wyeth Rotashield® & Simio-humano & Retirada \\
WC-3-humano & G1-G4, G6 P1A[8], P7[5] & MSD RotaTeq ${ }^{\circledR}$ & Bovino-humano & Licenciada \\
UK-humano & G1-G4, P7[5] & NIH & Bovino-humano & Fase II \\
\hline
\end{tabular}

*Institución con la cual se identifica el desarrollo de la vacuna; no corresponde necesariamente a la institución en donde se originó la cepa vaccinal. 
en el concepto de inmunidad heterotípica que supone que la protección conferida por la vacuna permitirá proteger contra rotavirus humano natural aunque su tipo VP7 y/o VP4 sean diferentes a la vacuna. La protección contra rotavirus de serotipos diferentes a la vacuna podría estar conferida por la inmunidad inducida contra el componente VP4, en el caso de ser compartido, y/o contra otros epítopes virales no reconocidos a la fecha. De esta manera se desarrollaron las primeras vacunas, basadas en rotavirus obtenidos de vacunos, simios y oveja (Tabla 1). Los estudios de eficacia con estas vacunas no fueron consistentes; se demostró una eficacia protectora aceptable en Finlandia pero no en países en desarrollo. Esta estrategia de desarrollo se ha abandonado y ha sido reemplazada por el uso de virus de procedencia humana y de virus reordenados. Sin embargo, debe mencionarse que existe una vacuna licenciada y en uso en China, con cepa originaria de oveja, producida por el Instituto Linzhou, de la cual no se tiene mayor información científica.

Las vacunas monovalentes humanas se basan en el concepto de que un rotavirus obtenido de un ser humano protegerá más eficientemente contra infección que un virus obtenido de un animal. Esta estrategia es la base de la vacuna "Rotarix ®" que se discutirá más adelante, desarrollada por Glaxo SmithKline Biologicals (GSK) ${ }^{35}$. Esta cepa de serotipo P1A[8]G1, el serotipo de mayor prevalencia mundial, fue obtenida de un niño y atenuada con multiples pasajes en el laboratorio. Rotavirus obtenidos de recién nacidos asintomáticos de variados serotipos (Tabla 1) son candidatos a vacunas encontrándose en diferentes fases de desarrollo. La posibilidad de que estos candidatos lleguen a ser licenciados a futuro requerirá de estudios de eficacia y seguridad semejantes a los ya realizados por GSK y Merck Sharp \& Dohme (MSD) para las vacunas Rotarix ${ }^{\circledR}$ y Rotateq ${ }^{\circledR}$ respectivamente.

Las vacunas multivalentes mantienen el concepto de utilizar un virus animal como base (virus simio o bovino) modificado en su VP7 y/o VP4 mediante reordenamiento genético (Figura 1). Reordenamiento se refiere al virus resultante de la coinfección celular por dos virus diferentes. Producto de esta coinfección se producirán virus en el que existe una "mezcla" de segmentos genéticos de ambos virus parentales, en la medida que estos virus reordenados son viables. Los virus seleccionados son quimeras que incluyen 10 segmentos genéticos del virus animal original y un segmento, correspondiente a aquel que codifica para la proteina VP7 o VP4 del virus humano. De esta manera las vacunas multivalentes incluyen a cuatro o cinco virus reordenados antigenicamente diferentes, cubriendo así los serotipos predominantes, G1, G2, G3, y G4 en caso de Rotashield ${ }^{\circledR}$ de Wyeth Laboratorios, y G1, G2, G3, G4 y P8 en el caso de Rotateq ${ }^{\circledR}$ de MSD (Tabla 1).

\section{Experiencia con Rotashield $\AA$}

La vacuna multivalente en la Figura 1, recombinante simio-humana de Wyeth Lederle, fue la primera vacuna anti-rotavirus licenciada en el mundo, en el año 1998. Esta vacuna fue ampliamente estudiada por más de 10 años en diferentes regiones del globo ${ }^{29-31,33,34}$. En resumen se puede señalar que la protección anti-rotavirus conferida con esta vacuna fue cercana a $50 \%$ contra todo tipo de infección, de $80 \%-95 \%$ contra infección moderada a grave y de $75 \%$ a $100 \%$ contra hospitalización por diarrea con deshidratación. Los estudios de Fase II y III sugirieron fuertemente que la vacuna era segura, aunque se detectó una tendencia, no significativa, a desarrollarse mayor número de casos de invaginación intestinal en niños vacunados comparado con niños que recibieron placebo; ocurrieron 5 casos de invaginación en 10.054 vacunados versus un caso en 4.633 controles. La vacuna fue aprobada para su uso en E.U.A. en 1998 y apoyada por la Academia Americana de Pediatría. Sin embargo, en 1999 se dieron a conocer los resultados de un estudio caso-control realizado en múltiples estados de E.U.A. por el Rotavirus Vaccine Field Investigation Team, del Centers for Disease Control and Prevention, que demostraron un aumento de incidencia de invaginación intestinal en niños vacunados. En aproximadamente 600.000 niños que recibieron la vacuna se detectó un aumento de invaginación intestinal, especialmente entre los días 3 y 7 post vacunación, primordialmente de la primera dosis, y en menor grado de la segunda. La estimación actual del riesgo de invaginación atribuible a la vacuna Rotashield ${ }^{\circledR}$ es cercano a 1:10.000 vacunados ${ }^{37-42}$. Estos resultados llevaron a suspender el uso de la vacuna tetravalente recombinante Rotashield ${ }^{\circledR}$ en E.U.A., y su retiro del mercado por parte de Wyeth Lederle asestando un duro golpe a la posibilidad de contar con una vacuna anti-rotavirus.

Las razones para este raro evento adverso con Rotashield® no están claramente dilucidadas. Estudios recientes han permitido determinar que invaginación intestinal ocurre en uno de cada 1.000 a 2.000 niños bajo 2 años de edad por causas no precisadas ${ }^{43-47}$. El porqué Rotashield ${ }^{\circledR}$ se asoció en una pequeña proporción de niños a esta grave afección es a la fecha materia de 
especulación. Una posibilidad es que esta vacuna compuesta por virus con proteínas propias de virus que infectan a simios y humanos, hubiese despertado una respuesta intestinal anómala en un pequeño número de lactantes predispuestos a desarrollar invaginación intestinal (teoría del gati1lo).

\section{Las nuevas vacunas}

Dos compañías farmacéuticas, en conjunto con investigadores de todo el mundo, apoyados por organismos internacionales y organismos no gubernamentales, tomaron el desafío de seguir adelante con el desarrollo de nuevas vacunas anti-rotavirus (Tabla 2). Este desafío era mayúsculo porque junto con demostrar adecuada eficacia, las nuevas vacunas deberían asegurar su no asociación con invaginación intestinal. Ello requeriría de estudios de campo con al menos 60.000 niños enrolados.

La vacuna pentavalente Rotateq ${ }^{\circledR}$ de MSD ha completado su estudio de Fase III realizado en fundamentalmente en Finlandia y E.U.A., con participación menor de otros cinco países incluyendo Latinoamérica. La publicación de este estudio estará disponible durante el año 2005. Presentaciones preliminares han demostrado lo siguiente ${ }^{48-50}$ :

- En cerca de 70.000 niños participantes, la mitad de los cuales recibió vacuna y la mitad placebo, ocurrieron 12 casos de invaginación intestinal en vacunados y 15 en el grupo placebo en el seguimiento hasta el año de edad. En el período más crítico correspondiente a la ventana de 42 días después de cualquiera de las tres dosis ocurrieron 6 casos en vacunados y 5 en el grupo placebo. La distribución temporal de casos de invaginación fue igual en ambos grupos; no se observó una concentración exagerada de casos después de cualquier dosis de vacuna. La conclusión es que Rotateq ${ }^{\circledR}$ no tiene asociación con invaginación intestinal.

- Para la cohorte completa de 69.274 niños, las hospitalizaciones y visitas de urgencia por rotavirus disminuyeron en 96 y $93 \%$ en vacunados, en comparación con el grupo placebo. Un total de 5.686 niños fue seguido para determinar eficacia de la vacuna. La protección reportada contra gastroenteritis por rotavirus con cualquier grado de severidad fue de $74 \%$ y contra gastroenteritis grave, usando el score clínico de Rennels, de 98\%. La reducción de las visitas médicas fue de $86 \%$.

- El tamaño de muestra utilizado permitió demostrar protección significativa contra infección por los serotipos G1, (75\%, IC 95\%: 67$88 \%)$ y $\mathrm{G} 2,(63 \%$, I.C. $95 \%$ : $2,6-88 \%)$. No se pudo demostrar protección contra G3 (vacunados 1, placebo 6), G4 (vacunados 3, placebo 6), ni G9 (vacunados 1, placebo 3).

Rotarix ${ }^{\circledR}$ es una vacuna monovalente obtenida de un virus vivo atenuado, la cepa 89-12 desarrollada por Richard Ward y David Bernstein del Gamble Institute de E.U.A. La cepa de serotipo P1A[P8]G1 fue adquirida por GSK Biologicals que la sometió a nuevos procedimientos de atenuación. El concepto fundamental detrás de esta vacuna es que dos dosis proveerán protección contra rotavirus naturales de tipo G1, G3, G4 y G9 y posiblemente G2. La vacuna se está aplicando en México desde mediados del 2005 y ha sido aprobada para uso en Brasil, Argentina, y Chile a la fecha, y próximamente en el resto de America Latina, África y Europa. Se administra en forma oral (formula liofilizada) diluida con buffer. La primera dosis se puede administrar a las 6-14 semanas de edad y la segunda a las 14-24 semanas,

Tabla 2. Características de vacunas anti-rotavirus licenciadas

\begin{tabular}{lll}
\hline Características & Rotarix® & RotaTeq® \\
\hline Productor & GlaxoSmithKline Biologicals & Merck Sharp \& Dohme \\
$\mathrm{N}^{\circ}$ serotipos & Monovalente & Pentavalente \\
Serotipos incluidos & $\mathrm{P} 1 \mathrm{~A}[8] \mathrm{G} 1$ & $\mathrm{P} 7[5] \mathrm{G} 1 \mathrm{P} 7[5] \mathrm{G} 2$, \\
& & $\mathrm{P} 7[5] \mathrm{G} 3 \mathrm{P} 7[5] \mathrm{G} 4$, \\
& & $\mathrm{P} 1 \mathrm{~A}[8] \mathrm{G} 7$ \\
Formulación & Monodosis, liofilizada, reconstituir con buffer & Monodosis, líquida \\
$\mathrm{N}^{\circ}$ dosis & Dos & Tres \\
Edad de administración & 2 y 4 meses & 2,4 y 6 meses \\
\hline
\end{tabular}


con un intervalo mínimo entre dosis de 4 semanas. La vacuna se puede co-administrar con vacunas regulares del Programa Ampliado de Inmunizaciones y otras vacunas extraprogramáticas.

La evidencia científica que apoya las indicaciones señaladas arriba se basa en 10 estudios de fases I y II desarrollados en Canadá, Europa, Asia, Africa, y Latinoamérica. En estos estudios se enrolaron 7.068 infantes (5.288 vacuna RV y 1.780 placebo) con más de 11.000 dosis administradas ${ }^{35,36,51-52}$. Los objetivos de estos estudios fueron evaluar seguridad y reactogenicidad, inmunogenicidad y eficacia con distintas dosis, diferentes edades de administración, con y sin vacunas concomitantes, con y sin lactancia materna. De estos estudios podemos resumir los siguientes resultados y conclusiones:

- Dos dosis de Rotarix ${ }^{\circledR}$, en el esquema indicado, presentan una adecuada respuesta inmune (IgA sérica), superior a 60\%. La co-administración con otras vacunas incluyendo polio oral a los dos y cuatro meses de edad no se asocia con una disminución de la seroconversión tanto para rotavirus como para las otras vacunas (DTP, polio, Hib) al cabo de la compleción de las dosis.

- La vacuna ha demostrado ser de muy baja reactogenicidad, sin diferencia con placebo.

- La eficacia al primer año de la vacuna en dos estudios de campo (Finlandia y Latinoamérica) demostró protección global contra diarrea por rotavirus de 73 y $70 \%$ y contra diarrea grave de 90 y $86 \%$. La protección contra hospitalización asociada a rotavirus fue de $79 \%$ en el estudio latinoamericano. El estudio finés demostró que la protección a dos años fue de $73 \%$ contra toda diarrea y $83 \%$ contra diarrea grave.

- En el estudio latinoamericano se pudo evaluar protección contra serotipos G1 y no G1 que representaron la mitad de los casos. La protección contra diarrea grave por serotipos no G1 (la gran mayoría era P1A[8]G9) fue de $83 \%$.

- Los estudios de Fase II demostraron igual riesgo de invaginación intestinal entre vacunados y niños que recibieron placebo (3 casos en 9.670 dosis de vacuna y un caso en 3.600 dosis de placebo para una incidencia de $0,06 \%$ en ambos grupos).

- El estudio de Fase III con más de 63.000 niños enrolados se realizó en 11 países latinoamericanos y en Finlandia durante el año 2003 y 2004, con el objetivo primario de determinar la seguridad de la vacuna ${ }^{53,54}$. Los casos de invaginación intestinal ocurridos hasta los 6 meses de edad fueron 16/31.673 receptores de placebo y de 9/31.552 vacunados para un riesgo relativo de $0,56(95 \% \mathrm{CI}: 0,25 ; 1,24)$. Durante la ventana de 30 días después de las dosis 1 y 2 ocurrieron 7 casos en el grupo placebo y 6 en niños vacunados. Se demostró así en forma concluyente que Rotarix ${ }^{\circledR}$ no se asocia con invaginación intestinal. Los niños vacunados tuvieron un número significativamente menor de hospitalizaciones y eventos adversos graves, dado fundamentalmente por la disminución de casos graves de gastroenteritis.

- La protección global contra gastroenteritis grave definida por dos criterios (necesidad de rehidratación y por el score clínico de Vesikari) fue de $85 \%$. La protección contra hospitalización por rotavirosis fue de $85 \%$ destacando que la protección contra hospitalización por toda diarrea aguda, independiente de su etiología, fue de $42 \%$. Esta cifra supera lo esperado considerando el $85 \%$ de protección conferida contra las hospitalizaciones por rotavirus solamente, hallazgo que sugiere una incidencia de diarrea por rotavirus mayor que la detectada por los métodos de ELISA usados en el estudio. Esta cifra de protección es de gran valor en una perspectiva de salud pública.

- En este estudio se siguió a una cohorte de 20.000 niños hasta el año de edad. Ello permitió determinar protección significativa contra los serotipos G1 (91\%, IC 95\% 72;92\%), G3 (88\%, IC 95\% 8\%; 100\%), y G9 (91\% IC $95 \% 62 \%, 99 \%$ ). Para G2 hubo una reducción no significativa de $41 \%$ (6 casos en vacunados y 10 en placebo). Hubo sólo 3 casos de infección grave por G4 lo cual no permitió determinar protección para este serotipo.

\section{Conclusiones y futuro}

El camino de desarrollo de vacunas antirotavirus ha sido largo y tortuoso. Los esfuerzos han dado resultado y el mundo ve hoy el nacimiento de dos nuevas vacunas avaladas por estudios sólidos de gran envergadura. Tanto Rotateq ${ }^{\circledR}$ como Rotarix ${ }^{\circledR}$ han demostrado ser seguras y conferir protección significativa contra los serotipos de rotavirus de mayor prevalencia en el mundo. Se requerirá mayor experiencia y estudio para determinar la protección contra serotipo G2 de ambas vacunas. Nuevos estudios están en marcha e irán aportando nueva información durante los próximos años. Rotarix ${ }^{\circledR}$ se puede administrar en forma concomitante con la vacuna anti-polio oral lo que facilita su incorporación a 
programas de vacunación en países en desarrollo. Rotateq ${ }^{\circledR}$ requiere aún de estudios necesarios para asegurar la no interferencia con vacuna antipolio oral.

La incorporación de estas vacunas en los programas ampliados de inmunizaciones de los países en desarrollo requerirá de importantes esfuerzos conjuntos de los gobiernos, los laboratorios productores, organismos internacionales, no gubernamentales y fundaciones. El impacto potencial de estas vacunas en la disminución de la morbimortalidad infantil es claro y significativo, especialmente en los países más pobres del mundo. Debe comenzar a la brevedad una discusión seria, con participación de todos los actores relevantes, basada en estudios de costo-efectividad en los países en desarrollo para que la introducción de estas vacunas ocurra más temprano que tarde. En los países industrializados las vacunas anti-rotavirus disminuirán en forma importante las consultas médicas y hospitalizaciones por diarrea aguda, por lo que su uso deberá también ser analizado en profundidad por sus autoridades sanitarias. La introducción de estas vacunas deberá acompañarse de sistemas de vigilancia centinela tanto para monitorizar su seguridad y su impacto en la disminución de enfermedad y en la dinámica de circulación de serotipos. Este requisito, necesario para toda nueva vacuna en un mundo moderno, permitirá responder en forma oportuna a cualquier evento no esperado así como a cambios epidemiológicos/virológicos hoy no vislumbrados, que pudieran requerir a futuro de modificaciones de las vacunas o de los esquemas vaccinales.

\section{Resumen}

Rotavirus es la primera causa de diarrea aguda grave en niños bajo 3 años de edad en el mundo. Esta infección es responsable de 25 millones de consultas, 2 millones de hospitalizaciones y 440.000 muertes por año en niños con menos de 5 años. El desarrollo de vacunas anti-rotavirus ha sido un camino largo y tortuoso marcado por la abrupta caída de Rotashield® en 1999 debido a su asociación con invaginación intestinal. Luego de seis años de intensa investigación, el mundo celebra la licencia de dos nuevas vacunas que, a pesar de ser diferentes en su formulación y forma de administración, han demostrado ser seguras y no asociadas a invaginación intestinal, en estudios de Fase III de gran magnitud, que enrolaron más de 60.000 niños. Estas dos vacunas, Rotarix ${ }^{\circledR}$ de Glaxo SmithKline Biologicals y Rotateq ${ }^{\circledR}$ de Merck Sharp \& Dohme son altamente eficaces contra diarrea grave causada por rotavirus de los serotipos más prevalentes en el mundo. La incorporación de estas vacunas, más temprano que tarde, especialmente en los países más pobres del mundo, requerirá de un esfuerzo conjunto de los gobiernos, laboratorios productores, organismos internacionales y no gubernamentales y fundaciones de beneficencia.

\section{Referencias}

1.- Parashar U D, Bresee J S, Glass R I. The global burden of diarrheal disease in children. Bull World Health Organ 2003: 81: 236.

2.- Parashar U D, Hummelman E G, Bresee J S, Miller M A, Glass R I. Global illness and deaths caused by rotavirus disease in children. Emerg Infect Dis 2003: 9: $565-72$

3.- Kosek M, Bern C, Guerrant R L. The global burden of diarrhoeal disease, as estimated from studies published between 1992 and 2000. Bull World Health Organ 2003: 81: 197-204.

4.- Prado V, O'Ryan M. Acute gastroenteritis in Latin America. Infect Dis Clin North Am 1994: 8: 77-106.

5.- O'Ryan M, Pérez-Schael I, Mamani N, Peña A, Salinas B, González G, et al. Rotavirus-associated medical visits and hospitalizations in South America: A prospective study at three large sentinel hospitals. Pediatr Infect Dis J 2001; 20: 685-93.

6.- O'Ryan M, Prado V, Pickering L K. A millennium update on pediatric diarrheal illness in the developing world. Semin Pediatr Infect Dis 2005; 16: 125-36.

7.- Matson D O, O'Ryan ML, Jiang X, Mitchell D K. Rotavirus, enteric adenoviruses, caliciviruses, astroviruses, and other viruses causing gastroenteritis. In Clinical Virology Manual, 3rd Edition. S Spector, RL Hodinka, SA Young, Editors. Washington DC; ASM Press, 2000, pp. 270-294.

8.- Gaggero A, O`Ryan M, Noel J, Glass R, Monroe S, Mamani N, et al. Prevalence of astrovirus infection among Chilean children with acute gastroenteritis. J Clin Microbiol 1998; 36: 3691-3.

9.- Velázquez F R, Matson D O, Calva J J, Guerrero L, Morrow A L, Carter-Campbell S, et al. Rotavirus infection in infants as protection against subsequent infections. N Engl J Med 1996; 335: 1022-8.

10.- Chiappini E, Azzari C, Moriondo M, Galli L, de Martino M. Viraemia is a common finding in immunocompetent children with rotavirus infection. J Med Virol 2005; 76: 265-7.

11.- Bishop R F, Davidson G P, Holmes I H, Ruck B J. Virus particles in epithelial cells of duodenal mucosa from children with viral gastroenteritis. Lancet 1973; 1: 1281-3.

12.- Estes M K. Rotaviruses and their replication. In: Fields BN, Knipe DM, Howley PM, eds. Fields Virology. 3rd ed. vol 2. New York: Raven Press, 1996, pp 16255573.

13.- O'Ryan M L, Matson D O, Estes M K, Pickering L K. Molecular epidemiology of rotavirus in children attending day care centers in Houston. J Infect Dis 1990; 162: 810-16.

14.- O’Ryan M L, Matson D O, Estes M K, Pickering L $\mathrm{K}$. Anti-rotavirus $\mathrm{G}$ type-specific and isotype antibodies in children with natural rotavirus infections. J Infect Dis 1994; 169: 504-11.

15.- Matson D O, O'Ryan M, Pickering L K, Chiba S, Nakata S Raj P, et al. Characterization of serum 
antibody responses to natural rotavirus infections in children by VP7-specific epitope-blocking assays. J Clin Microbiol 1992; 30: 1056-61.

16.- Offit P A. Host factors associated with protection against rotavirus disease: The skies are clearing. J Infect Dis 1996174 (Suppl 1): S59-64.

17.- Chiba S, Yokoyama T, Nakata S, Morita Y, Urasawa T, Tanigushi K, et al. Protective effect of naturally acquired homotypic and heterotypic rotavirus antibodies. Lancet 1986; 1: 417-21.

18.- Gentsch J R, Woods P A, Ramachandran M, Das B K, Leite J P, Alfieri A, et al. Review of G and P typing results from a global collection of rotavirus strains: implications for vaccine development. J Infect Dis 1996; 174 (Suppl 1): S30-6. Review.

19.- Unicomb L E, Podder G, Gentsch J R, Woods P A, Hasan K Z, Faruque A S, et al. Evidence of highfrequency genomic reassortment of group A rotavirus strains in Bangladesh: Emergence of type G9 in 1995. J Clin Microb 1999; 37: 1885-91.

20.- Banyai K, Gentsch JR, Schipp R, Jakab F, Meleg E, Mihaly I, et al. Dominating prevalence of P[8],G1 and P[8],G9 rotavirus strains among children admitted to hospital between 2000 and 2003 in Budapest, Hungary. J Med Virol 2005; 76: 414-23.

21.- Rahman M, Matthijnssens J, Goegebuer T, De Leener K, Vanderwegen L, van der Donck I, et al. Predominance of rotavirus G9 genotype in children hospitalized for rotavirus gastroenteritis in Belgium during 1999-2003. J Clin Virol 2005 ; 33: 1-6

22.- O’Ryan M L, Mamani N, Avendaño L F, Cohen J, Pena A, Villarroel J, et al. Molecular epidemiology of human rotaviruses in Santiago, Chile. Pediatr Infect Dis J 1997; 16: 305-11.

23.- Ramachandran M, Das B K, Vij A, Kumar R, Bhambal S $\mathrm{S}$, Kesari N, et al. Unusual diversity of human rotavirus $\mathrm{G}$ and $\mathrm{P}$ genotypes in India. J Clin Microb 1996; 34 : 436-9.

24.- Santos N, Lima R C, Pereira C F, Gouvea V. Detection of rotavirus types G8 and G10 among Brazilian children with diarrhea. J Clin Microbiol 1998; 36: 2727-9.

25.- Kilgore P E, Unicomb L E, Gentsch J R, Albert M J, Mcelroy C A, Glass R I. Neonatal rotavirus infection in Bangladesh strain characterization and risk factors for nosocomial infection. Pediatr Infect Dis J 1996; 15: 672-7.

26.- Tam J S, Zeng, B J, Lo S K, Yeung, C Y, Lo M, Ng M $\mathrm{H}$. Distinct population of rotaviruses circulating among neonates and older infants. J Clin Microbiol 1990; 28: 1033-8.

27.- Palombo E A, Bishop R F. Genetic and antigenic characterization of a serotype G6 human rotavirus isolated in Melbourne, Australia. J Med Virol 1995; 47: 348-54.

28.- Bishop R F, Barnes G L, Cipriani E, Lund J S. Clinical immunity after neonatal rotavirus infection. A prospective longitudinal study in young children. N Engl J Med 1983; 309:72-6.

29.- Santosham M, Moulton L H, Reid R, Croll J, Weatherholt H, Ward R, et al. Efficacy and safety of high-dose rhesus-human reassortant rotavirus vaccine in native American populations. J Pediatr 1997; 131: 632-8.

30.- Pérez-Schael I, Guntiñas M, Pérez M, Pagone V, Rojas A M, González R, et al. Efficacy of the rhesus-based quadrivalent vaccine in infants and young children in Venezuela. N Engl J Med 1997; 337: 1181-7.
31.- Joensuu J, Koskenniemie E, Pang X L, Vesikari T. Randomised placebo-controlled trial of rhesus-human reassortant rotavirus vaccine for prevention of severe rotavirus gastroenteritis. Lancet 1997; 350:1205-9.

32.- Estes M K. Advances in molecular biology: Impact on rotavirus vaccine development. J Infect Dis 1997; 174 (Suppl 1): S37-46.

33.- Rennels M B, Glass R I, Dennehy P H, Bernstein D I, Pichichero M I, Zito E T, et al. Safety and efficacy of high-dose rhesus-human reassortant rotavirus vaccines: report of the national multicenter trial. Pediatrics1996; 97: 7-13.

34.- Kapikian A Z, Hoshino Y, Chanock R M, PérezSchael I. Efficacy of a quadrivalent rhesus rotavirusbased human rotavirus vaccine aimed at preventing severe rotavirus diarrhea in infants and young children. J Infect Dis 1996; 174 (Suppl 1): S65-72.

35.- De Vos B, Vesikari T, Linhares AC, Salinas B, PérezSchael I, Ruiz-Palacios GM, et al. A rotavirus vaccine for prophylaxis of infants against rotavirus gastroenteritis. Pediatr Infect Dis J 2004 ; 23: S17982.

36.- Vesikari T, Karvonen A, Korhonen T, Espo M, Lebacq E, Forster J, et al. Safety and immunogenicity of RIX4414 live attenuated human rotavirus vaccine in adults, toddlers and previously uninfected infants. Vaccine 2004; 22: 2836-42.

37.- Centers for Disease Control and Prevention. Intussusception among recipients of rotavirus vaccine - United States, 1998-1999. MMWR Morb Mortal Wkly Rep 1999; 48: 577-81.

38.- Centers for Disease Control and Prevention. Withdrawal of rotavirus vaccine recommendation. MMWR Morb Mortal Wkly Rep 1999; 48: 1007.

39.- Murphy T V, Gargiullo P M, Massoudi M S, Nelson D $\mathrm{B}$, Jumaan A O, Okoro C A, et al. Intussusception among infants given an oral rotavirus vaccine. $\mathrm{N}$ Engl J Med 2001; 344: 564-72.

40.- Kramarz P, France E K, Destefano F, Black S B, Shinefield H, Ward J I, et al. Population-based study of rotavirus vaccination and intussusception. Pediatr Infect Dis J 2001; 20: 410-6.

41.- Murphy B R, Morens D M, Simonsen L, Chanock R M, La Montagne J R, Kapikan A Z. Re-appraisal of the association of intussusception with the licensed live oral rotavirus vaccine challenges initial conclusions. J Infect Dis 2003; 187: 1301-8.

42.- Peter G, Myers M. National Vaccine Advisory Committee. National Vaccine Program Office. Intussusception, rotavirus, oral vaccines: summary of a workshop. Pediatrics 2002; 110: 67.

43.- Lucero Y, Valenzuela M T, O'Ryan M. Clinical and epidemiological profile of intestinal intussusception among infants of Metropolitan Santiago. Rev Méd Chile 2004; 132: 565-72.

44.- O'Ryan M, Lucero Y, Pena A, Valenzuela MT. Two year review of intestinal intussusception in six large public hospitals of Santiago, Chile. Pediatr Infect Dis J 2003; 22: 717-21.

45.- Abate H, Linhares A C, Venegas G, et al. A multicenter study of intussusception in Latin America: first year results. Paper presented at International Congress of Pediatrics; August 15-20, 2004; Cancun, Mexico.

46.- Pérez-Schael I, Escalona M, Salinas B, Materan M, Pérez M E, González G. Intussusception-associated hospitalization among Venezuelan infants during 1998 through 2001: anticipating rotavirus vaccines. Pediatr Infect Dis J 2003; 2: 234-9. 
47.- Saez-Llorens X, Guevara J N. Intussusception and rotavirus vaccines: what is the background risk? Pediatr Infect Dis J 2004; 23: 363-5.

48.- Itzler R, Matson D, Vesikari T, Coia M, Cook J, Davies $\mathrm{G}$, et al. The effectiveness of a pentavalent (human-bovine) reassortant rotavirus vaccine (PRV) to reduce hospitalizations and emergency department (ED) visits for rotavirus gastroenteritis. Pediatric Academic Societies' 2005 Annual Meeting: Washington, DC; May 14-17, 2005.

49.- Vesikari T, Matson D, Van Damme P, Heyse J, Dallas M, Goveia M, et al. Incidence of intussusception with the pentavalent (human-bovine) reassortant rotavirus vaccine (PRV) is similar to placebo. 23rd Annual Meeting of the European Society for Pediatric Infectious Diseases: Valencia, Spain; May 18-20, 2005.

50.- Vesikari T, Matson D, Dennehy P, Santosham M, Rodriguez Z, Goveia M, et al. Protection against rotavirus gastroenteritis of multiple serotypes by a pentavalent (human-bovine) reassortant rotavirus vaccine (PRV). 23rd Annual Meeting of the European Society for Pediatric Infectious Diseases: Valencia, Spain; May 18-20, 2005.

51.- Bernstein DI, Sack DA, Reisinger K, Rothstein E, Ward
RL. Second year follow-up evaluation of live attenuated human rotavirus vaccine $89-12$ in healthy infants. J Infect Dis 2002; 186: 1487-9.

52.- Bernstein DI, Sack DA, Rothstein E, Reisinger K, Smith VE, O'Sullivan D, et al. Efficacy of live attenuated human rotavirus vaccine 89-12 in infants. Lancet 1999; 354: 287-90.

53.- Vesikari T, Karvonen A, Puustinen L, Zeng SQ, Szakal SD, Delem A, et al. Efficacy of RIX4414 live attenuated human rotavirus vaccine in Finnish infants. Pediatr Infect Dis J 2004; 23: 937-43.

54.- O'Ryan M, Lanata C, Abate H, Pérez Schael I, Rivera $\mathrm{M}$, Ortega $\mathrm{E}$, et al. A novel rotavirus vaccine RIX4414 is not associated with intussusception. $44^{\text {th }}$ International Conference on Antimicrobials Agents and Chemotherapy-ICAAC, Washington D.C., U.S.A., October 30-November 2, 2004.

55.- Velásquez R, Abata H, Costa-Clemens S A, Espinoza F, Gillards P, Linhares A C, et al. The human monovalent $\mathrm{G} 1 \mathrm{P}[8]$ rotavirus vaccine, Rotarix ${ }^{\mathrm{TM}}$ is highly efficacious and provides cross-protection against G1 and non-G1 serotypes. 23rd Annual Meeting of the European Society for Pediatric Infectious Diseases: Valencia, Spain; May 18-20, 2005.

Correspondencia a:

Miguel O'Ryan Gallardo

moryan@med.uchile.cl 